\title{
Unmanned Aerial Vehicles (UAVs)-An Overview
}

\author{
Dhvani Ajmera and Meet Saroliya \\ Diploma in Computer Engineering Shri Bhagubhai Mafatlal Polytechnic, Mumbai, \\ India studentdhvani@gmail.com
}

\begin{abstract}
Unmanned Aerial Vehicles (UAVs), which have uses in research, business, and public service, have recently emerged as a crucial component of the military's operating environment. Drones of various varieties are being created to fulfil the demands of various users. As a result, the study topics in the UAV domain are expanding as the types and numbers of UAVs increase. In the recent years, the field of unmanned aerial vehicles has exploded. The nature of the study has been grouped into many groups, as well as an overview of existing research fields in the UAV sector. This category encompasses two types of studies: technical and operational research. The two categories of technical research are on-board and ground technologies. This overview is intended to serve as a starting point for beginners to the domain, guiding them in determining where to focus their research, identifying relevant research fields, and focusing on the correct topics.
\end{abstract}

Key words - UAV (Unmanned Aerial Vehicle), Neural Net- works, Deep Learning, Artificial Intelligence, Machine Learning

\section{INTRODUCTION}

DRONE is an acronym for Dynamic Remotely Operated Navigation Equipment, which is a type of unmanned aerial vehicle is a plane without a human pilot that is controlled remotely or autonomously by computers. Drone firms like DJI, Parrot, 3DRobotics have been quickly growing in recent years, thanks to the ongoing development of associated technology. Drones have been widely deployed in both military and civilian domains, and their consumption has exploded due to their low cost and user-friendly operation. Drone purchases totalled 1.9 million in 2016, according to the Federal Aviation Administration (FAA), and are expected to reach 4.3 million by 2020 .

\section{HISTORY OF DRONE}

Unmanned aerial vehicle, commonly known as a DRONE (Dynamic Remotely Operated Navigation Equipment) is a plane that is flown remotely or autonomously by computers and does not have a human pilot. Due to the continual development of accompanying technology, drone companies such as DJI, Parrot, and 3DRobotics have seen rapid growth in recent years.

Drones have been widely used in both military and civilian applications, and their popularity has grown as a result of their low cost and easy operation. According to the Federal Aviation Administration (FAA), drone purchases totalled 1.9 million in 2016 and are predicted to reach 4.3 million by 2020 .

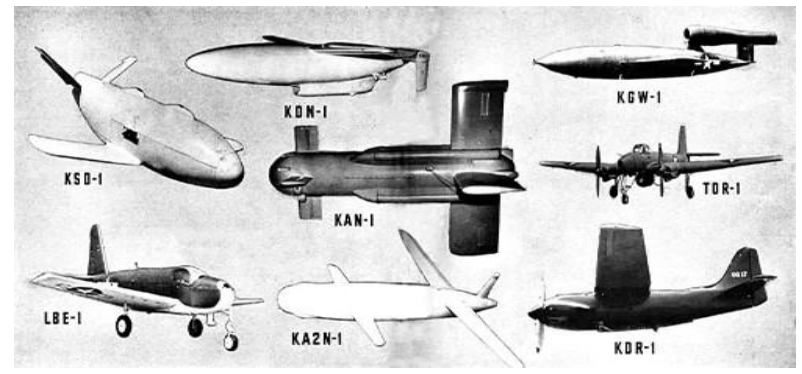

Fig. 1. History

\section{AI AND ML IN Unmanned AeRial Vehicles}

Drones are unmanned aerial vehicles (UAVs) that can perform a variety of activities. Initially, these devices were managed manually and remotely. Artificial intelligence is increasingly often used in drones, which automates part or all activities. Thanks to artificial intelligence, drone providers max gather and implement visual and environmental data utilising data from sensors attached to the drone[2,3]. This data allows for autonomous or assisted flying, which streamlines operations and increases accessibility. Drones have therefore become a component of the smart mobility services that are now commercially offered to companies and consumers. Drones with AI technology rely heavily on computer vision. Thanksto this technology, drones can detect objects while flying as well as analyse and collect data on the 
ground. In computer vision, a neural network is utilised to do high-performance onboard image processing. A layered design for implementing machine learning algorithms is a neural network. Drones are made feasible by technological advancements.

At greater speeds, drones become unstable, and it is hard to anticipate their direction, leading in collisions. Engineers at MIT have developed a new algorithm to help drones navigate over obstacles. This paves the way for drones to travel at least $20 \%$ faster and avoid mishaps than when taught using existingalgorithms,

\section{A. What is AI?}

Artificial intelligence is a branch of computer science that aims to create a computer system that can think like a person. It is formed from the terms "artificial" and "intelligence", which together signify "human-made thinking ability."

As a result, we may define it as, "Artificial intelligence is a technology using which we can create intelligent systems that can simulate human intelligence."

Artificial intelligence systems do not need to be preprogrammed; instead, they employ algorithms that function in conjunction with their own intellect. Reinforcement learning algorithms and deep learning neural networks are examples of machine learning algorithms. Siri, Google's AlphaGo, AI in chess, and other applications of AI are all examples.

\section{B. What is Machine Learning?}

Machine learning is about extracting knowledge from the data. It can be defined as,

"Machine learning is a sub-field of artificial intelligence, which enables machines to learn from past data or experiences without being explicitly programmed."

Without being explicitly coded, machine learning allows a computer system to generate predictions or make judgments based on past data. Machine learning makes use of a large quantity of structured and semi-structured data in order for a machine learning model to provide reliable results or make predictions based on it.

Machine learning is based on an algorithm that learns on its own with the use of previous data. It only works for restricted domains; for example, if we create a machine learning model to recognise dog photographs, it will only provide results for dog pictures; however, if we add fresh data, such as a cat picture, it will become unresponsive. Machine learning is utilised in a variety of applications, including online recommender systems, Google search engines, email spam filters, and Facebook auto friend tagging suggestions, among others.

\section{Key Components of A DRONe}

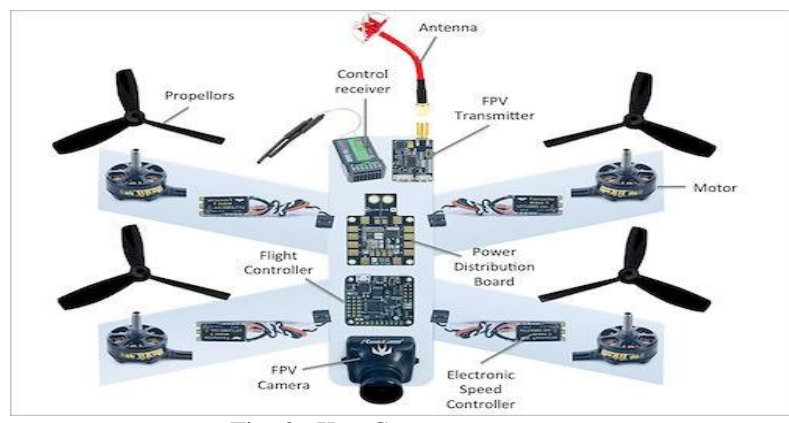

Fig. 2. Key Components

\section{TRENDS AND OPPORTUNITIES IN UAV}

Technology has advanced at a breakneck pace during the previous two decades. Drones were formerly thought to be a strange notion, but they are now being employed in a variety of applications. Drones' widespread use has spawned a multi billion-dollar business that is predicted to reach $\$ 12$ billion by the end of 2020. For investors, this industry gives a fantastic opportunity.

Drones, or unmanned aircraft systems, are flying devices that are fitted with sensors and GPS. They may be operated remotely using a computer or other device. After drones were brought to the market, the majority of people became aware of their use. Drones are being employed in business purposes as well as by enthusiasts.

Drones are also widely employed in military and defence applications, where they are used for reconnaissance, bombing attacks, and air support. In reality, the Pentagon just approved a $\$ 6$ billion budget for drone research and development.

According to a recent new update, Google and Amazon are working on their own drones to make it easier to transport packages by air. Facebook has offered another intriguing proposal, in which they are considering developing gigantic drones that can transport signal to remote regions for direct internet connection. Drones have become an important part of the film industry in today's world, and news reporters are using them to carry information from inaccessible locations.

\section{A. Current Trends}

1) Surveying and Mapping: Aerial surveying and mapping is one of the fastest-growing applications for drone technology. Unmanned aerial vehicles may now accomplish what used to require either foot treks or manned planes. This component 
of drone use has grown in popularity to the point that it now accounts for around $37 \%$ of the civilian drone industry growthin the previous two years.

2) Aerial Infrastructure Inspection: Because of the proliferation of pipelines and pipeline infrastructure, this field has grown significantly. Previously, if a corporation wanted to examine the condition of certain portions of the pipeline, it had to deploy employees on foot or hire a manned aircraft, both of which were expensive. Drones have enabled for a morecost-effective inspection approach. Drones aren't just used to inspect pipelines; they're also used to inspect agricultural land and railroads. Over the previous several years, aerial infrastructure inspection has accounted for $25 \%$ of the civiliandrone industry growth.

3) Transport and Warehousing Industry: The energy sector is the largest civilian drone market, but it is not the fastest expanding. Drones are largely used to repair and support pipeline infrastructure. The transportation and warehousingbusiness has that status.

4) China Will Become World's Largest UAV Market: The United States is now the world's largest drone market, closely followed by China. This is unlikely to remain, since the Chinese drone market is predicted to rise to 18.4 billion in 2024, compared to 11.4 billion in the United States.Military Largest Drone Application: Military contracts accounted for $75 \%$ of the total UV industry revenue in 2019 . This may come as a surprise to some. This suggests that the United States' defence budget is fueling drone sales. The United States has approved a budget of $\$ 6$ billion to be spent entirely on unmanned aerial vehicle (UAV) technology.

5) India Is The Largest Emerging Drone Market: Drones were not legalised in India until 2018, years behind the majority of other large countries. This implies that drone technology has just recently been introduced to one of the world's major marketplaces. India is anticipated to become the world's third-largest AV technology market by 2024, having already been the fastest-growing drone industry.

6) From 2019 to 2024, drone sales are expected to triple: Drones and other UAV technologies are expected to treble in sales by 2024 , according to unit sales data.

\section{APPLICATIONS OF UAV}

Drones have three main applications:

\section{A. SURVEILLANCE}

Drones may be equipped with a variety of surveillance technologies to gather HD video and still images at any time of day or night. Drones can be equipped with technology that allows them to listen in on phone calls, track GPS movements, and collect licence plate information. Because of the high payload compatibility, a range of surveying devices, including as lidar scanners, multi- and hyperspectral sensors, and more, may be used 24 hours a day with low labour and expenditures. Drone surveillance is the use of unmanned aerial vehicles (UAVs) to capture still photographs and video in order to acquire data on specified targets, which can be persons, organisations, or areas. Drone surveillance enables for the collecting of information about a target while remaining unnoticed from a distance or height. Drone surveillance enables for the stealth collecting of data about a target from a distance or at a high altitude. Drones, of course, aren't new.

\section{B. WEATHER FORECAST}

The world around us is changing. Natural calamities are similar in this regard. Drones, obviously, are not as accurate as satellite images in predicting severe weather occurrences. They are, nonetheless, capable of providing critical assistance in the case of a disaster. Government agencies and insurers are becoming increasingly aware of the potential for using them to estimate post-disaster damages, especially in areas where people have not been authorised as safe to access. Drones taking air samples are a major advance over traditional data gathering methods, and they have the potential to enhance the accuracy of weather forecasting models significantly. Is it really that important? Models that are more accurate have an influence on both the short and long term. It not only allows meteorologists to produce better 10-day weather forecasts, butit also allows them to provide more advanced warnings for phenomena like tornadoes and whether or not hurricanes will arrive. The bulk of our weather happens in the boundary layer,

which is the lowest layer of the atmosphere. A bewildering number of variables and forces determine the weather conditions that affect each of us. Attempting to determine what the weather will do next relies on very complex weather forecasting models, but the models' output is only as good as the data fed into them. Obtaining high-quality data is also more challenging than you would think. Drones that track the weather can assist with this. By flying through the whole vertical layer of the atmosphere's boundary layer, weather drones may collect important data on temperature, moisture, air pressure, and wind speed and direction. Weather drones may collect data using temperature, humidity, and air pressure sensors that are directly attached to the drone. Another way they collect data is by dropping sensors attached to parachutes from a high height. 


\section{DELIVERY}

There are several applications in which delivery drones are used. Health care: Medical supplies such as blood, immunizations, and pharmaceuticals, as well as other products such as drugs and medical samples, can be transported by drones. Drones are being used to transport blood donations, vaccines, medications, anti-venom treatments, organ transplants, and other medical supplies, which is gaining a lot of popularity. More than 30 companies, including drone service providers and internal services, employ drones to distribute medical sup-plies. Food: In addition to improving the aforementioned in- dustrial and commercial operations, delivery drones may now bring a lot of value to logistical supply chains. The prospect of having food delivered to you wherever you are through a drone has piqued people's curiosity in the benefits that delivery drones may bring to businesses in general and people's daily routines in particular. Drones have been suggested as a means of fast transporting prepared foods such as pizzas, tacos, and frozen drinks. Postal: When carrying packages, drones have a lower energy balance than normal delivery vehicles. In densely populated areas, drones consume a disproportionate amount of energy, and their range is significantly influenced by wind conditions. In rural regions, though, they may be able to compete with diesel-powered delivery vehicles. Drone testing has been done by postal companies in Australia, Switzerland, Germany, Singapore, the United Kingdom, and Ukraine to explore if unmanned delivery drone services are practical and lucrative.

Ship Resupply: Drones are being used to refuel offshore ships instead of smaller boats. Drones provide supplies to troops in the field, allowing for speedy delivery of emergency food and ammunition. The autonomous drones can carry 60 pounds of cargo and travel up to six kilometres.

\section{TYPES OF UAVS}

Drones can be categorized in a range of methods, including Drones for Photography, Drones for Aerial Mapping, Drones for Surveillance, and so forth[1]. The best classification of 'Drones', however, may be based primarily on aerial platforms. Drones are grouped into four types based on the type of its aerial platform use as follows:

- Multi Rotor Drones

- Fixed Wing Drones
- Single Rotor Drones

- Fixed Wing Hybrid VTOL

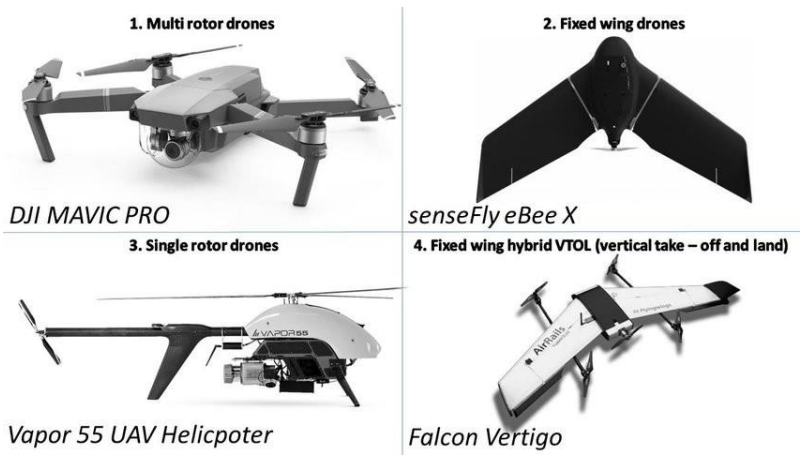

Fig. 3. Types of Drones

\section{A. Multi Rotor Drones}

Multi Rotor Drones are the most prevalent, and they are operated by both professionals and hobbyists. They're useful for a handful of tasks, including aerial photography and video surveillance. Multi-rotor drones are the easiest to develop and the cheapest alternative available among the four drone kinds (depending on aerial platform). Multi-rotor drones can be further classified based on the number of rotors on the platform into the following types: Tricopter (3 rotors), Quadcopter (4 rotors), Hexacopter (6 rotors), Octocopter (8 rotors). Out of these, Quadcopters are the most popular and widely used variant.

\section{B. Fixed Wings Drones}

Fixed-wing drones are not the same as multi-rotor drones in terms of design and construction. They have a wing, just like a regular aircraft. Fixed wing drones, unlike multi-rotor drones, never consume energy to stay afloat in the air (fixed wing drones can't stand still in air) resisting the gravity. Instead, they continue on their predetermined path or as directed by the guide control (potentially a human-operated remote unit) as long as their energy source allows.

\section{Single Rotor Helicopter}

Single-rotor drones resemble helicopters in terms of form and structure. Unlike a multi-rotor drone, a single-rotor model features only one large rotor and one small rotor on the drone's tail to control its direction. Drones with a single rotor are far 
more efficient than those with several rotors as they can fly for longer periods of time and are even powered by gas engines. In aerodynamics, the lower the number of rotors, the lower the object's spin. Quadcopters are far more stable than octocopters for this reason. Single-rotor drones are far more efficient than multi-rotor drones in this regard.

\section{Fixed Wing Hybrid VTOL}

These are hybrid models that combine the advantages of fixed-wing aircraft (longer flight time) with the advantages of rotor-based models (hover). This notion has been tested since the 1960s, but with little success. This notion has gainedfresh life and direction with the introduction of new generation sensors (gyros and accelerometers). Hybrid VTOLs combine automation with manual gliding. The drone is lifted into the air using a vertical lift from the ground. To keep the drone stable in the air, gyros and accelerometers work in an automated mode (autopilot concept). The drone is guided on the desired course via remote-based (or even programmed) manual control. There are some versions of this hybrid fixed wing models available in the market. However, the most popular one is drone used in Amazon commercials (for its Prime delivery service).

\section{Open Challenges in Future Directions}

\begin{tabular}{|c|c|c|}
\hline Issues & Major Challenges & Possible Solutions \\
\hline Privacy & Detection/Access to justice & $\begin{array}{l}\text { Hardware and software for device detection, } \\
\text { and data retention / Registry of owners and } \\
\text { devices }\end{array}$ \\
\hline Ownership & Accountability & $\begin{array}{l}\text { Registry of owners and devices/ Assign } \\
\text { liability for UAV owners/ }\end{array}$ \\
\hline Security & Control/Enforcement & $\begin{array}{l}\text { Creation of new infrastructure and } \\
\text { development of proper assets: UAV trackers } \\
\text { devices/automatic safe landing } \\
\text { Establishment of insurance entities etc. }\end{array}$ \\
\hline Regulatory & $\begin{array}{l}\text { Lack of comprehensive rules } \\
\text { and uniformity across } \\
\text { jurisdictions }\end{array}$ & $\begin{array}{l}\text { Redefinition of "reasonable expectation of } \\
\text { privacy"/ Definition of physical aerial } \\
\text { boundaries / Centralization of Powers }\end{array}$ \\
\hline $\begin{array}{l}\text { Business } \\
\text { Models }\end{array}$ & $\begin{array}{l}\text { Lack of clear guidelines to } \\
\text { operate in compliance with } \\
\text { the law }\end{array}$ & $\begin{array}{l}\text { Promote regulations for the development } \\
\text { UAV-related technologies }\end{array}$ \\
\hline
\end{tabular}

Fig. 4. Challenges

\section{LEADING COMPANIES INTO THE UAV INDUSTRY}

\section{A. DRONESENSE}

Location: Austin, Texas

DroneSense is a drone software platform for public safety professionals that transforms raw data gathered by drones into usable insights for police, fire, and other emergency response teams. The DroneSense OpsCenter allows several drone users to work together, see what each drone observes, and even tracka drone's flight path in real time.

DroneSense's public safety platform has been utilised by dozens of teams to tackle a range of threats to public safety. SWAT teams may use the Al-powered software to gather scene intelligence, analyse damage after storms and tornadoes, and even use thermal imaging to identify missing people.

\section{B. NEURALA}

\section{Location: Boston}

How it's using AI: Neurala is a deep learning neural network that assists drones in sifting through crowds to find and identify people of interest. It can also check and report onmassive industrial equipment, such as telephone towers, in real time. The business says that instead of taking hours or days to scan crowds for an individual, their Al-powered software just takes 20 minutes to interpret the image of an individual.

Industry impact: The Lindbergh Foundation uses Neuralapowered drones to combat elephant poaching in Africa. The artificially intelligent drones use the company's image recognition technology to monitor elephant herds and spot possible poachers miles before they reach the elephants.

\section{SCALE}

\section{Location: San Francisco}

How it's using Al: Scale employs artificial intelligence and machine learning to assist in the training of drones on aerial footage. Drones can use machine learning algorithms to recognise, classify, and map everything from small things like vehicles to entire neighbourhoods.

Industry impact: Insurance businesses like Liberty Mutual utilise the Scale machine learning platform for drone training. The UAVs are used to detect and quantify insurance claims

\section{Drone Detection Systems}

Drones used indiscriminately represent a significant danger to public safety and personal privacy[4]. Real-time detection of trespassing drones in sensitive locations is required. Drone detection technology is used to identify unmanned aircraft systems (UAS), or drones as they are more often known.Drone activity in the airspace is detected using a variety of methods, each with various capabilities based on the size of the drone and the detection range. Drones that communicate by radio frequency (RF) may be monitored using RF sensors, while those having GPS pre-programmed to a waypoint can be detected using radar. Pan, tilt, and zoom (PTZ) cameras can be 
used to record images of a drone and validate that it is a threat. Despite receiving broad attention in a range of civic and commercial purposes, Unmanned Air Vehicles (UAVs sometimes known as drones) offer a number of problems for airspace safety that may cause harm to people and property. In terms of the assailants' goals and sophistication, such threats can range from pilot inexperience to calculated attacks, but they all have the capacity to cause major disruption.

\section{SUMMARY}

Drones are unmanned aerial vehicles that can be utilised for a wide range of tasks, making their mark in sectors of military, civilian and commercial purposes. Drone providers can collect and implement visual and environmental data using data from sensors linked to the drone thanks to Artificial Intelligence and Machine Learning Algorithms. The UAVs can detect objects while flying, as well as analyse and collect data on the ground, thanks to this technology. The advancements in technology over the last two decades have been astounding such that most people became aware of the use of drones after they were introduced to the market. Drones that communicate by radio frequency can be traced with $\mathrm{F}$ sensors, while those that have GPS pre-programmed to a waypoint can be tracked with radar detection. With an accelerating growth in the field, therequirement of drone detection systems is also a must.

\section{CONCLUSION}

This paper discussed several essential terminologies related to drones, its applications as well as gave a brief view onthe emerging trends that we are to see in the years to come. UAVs are going to play a very important role in the future as it is already evident from today's times itself with the pace of the current advancements and hence this paper also covers the challenges that are faced as the usage of these aerial vehicles potentially increases, being it in a business or the military, leading to flexibility and convenience ensuring the growth of businesses in an efficient manner as well. Hence, future researchers should focus on addressing solutions tothese problems.

\section{ACKNOWLEDGMENTS}

The authors would like to express our humble gratitude and earnestly acknowledge the sincere efforts and valuable time taken out by our mentor, Mrs. Prachi A. Their valuable guid- ance and feedback have been a contribution in the completion of this research paper. We would also like to thank all our peers and everyone who motivated us and encouraged us to work on the paper.

\section{REFERENCES}

[1] https://www.circuitstoday.com/types-of-drones

[2] https://analyticsindiamag.com/ml-for-drones-better-faster-and-crashproof/

[3] https://www.wevolver.com/article/artificial-intelligence-in-dronetechnology

[4] https://riskandinsurance.com/drones-offer-risks-underwritingchallenges/ 